$$
\begin{aligned}
& \text { MPI-H } 94-2 \\
& \text { sw } 9409
\end{aligned}
$$

\title{
On Zero Modes and Gauge Fixing in Light-Cone Quantized Gauge Theories
}

\author{
A.C. Kalloniatis and H.C. Pauli \\ Max-Planck-Institut für Kernphysik \\ Postfach 103980 \\ D-69029 Heidelberg 1 \\ Germany
}

26 January, 1994

\begin{abstract}
Discretized Light-Cone Quantization potentially solves QCD in 3+1 dimensions. However the zero Fourier modes of bosonic fields are an outstanding formal problem. Here we study these zero modes of gauge bosons but only for QED in $3+1$ dimensions. Crucial to the present treatment is a set of gauge conditions which differ in different Fourier sectors of the theory. We shall specify in which of the sectors the conventional light-cone gauge $A^{+}=0$ is justified. In the new gauge setting almost all of the zero modes are not dynamical but constrained fields. Due to antiperiodic boundary conditions on the fermionic fields there are no fermion zero modes. But fermions complicate the non-perturbative structure of the constraints insofar as they generate coupled linear equations for the boson modes. Iterative solutions are given.
\end{abstract}

Preprint: MPIH-V2-1994

PQDQอ1291 


\section{Introduction}

Light-cone and the more recent discretized light-cone quantization (DLCQ) offer advantages not available in standard instant-form quantization for a non-perturbative treatment of gauge theories. These include kinematical Lorentz boosts [1] and a simpler vacuum structure, a broad introduction with complete references being given in ref. [2]. Due to the periodic boundary conditions or compactification onto a finite 'torus' geometry, the discrete momentum Fock space regulates the infrared region and is ready-made for numerical solution. The heart of this ambitious program is to give a controllable formulation of quantum field theory in terms of a Hamiltonian eigenvalue equation. The eventual target is nothing less than quantum chromodynamics and the strong interaction. Reasonably successful applications have been made to theories in one [3] as well as three space dimensions $[4,5,6]$. Nonetheless, it is now appreciated by the community that certain degrees of freedom have been mistreated at least at the formal level - namely the zero modes.

To use Dirac's nomenclature [1], the essential aspect which is responsible for all the differences between the 'front form' and the more familiar 'instant form' lies in the four dimensional Minkowski space D'Alembertian

$$
\square=\partial_{0}^{2}-\partial_{3}^{2}-\nabla_{\perp}^{2}=2 \partial_{+} \partial_{-}-\nabla_{\perp}^{2} .
$$

For more detail on our conventions we refer the reader to the appendix. In the instant form the D'Alembertian is always quadratic in the time derivative $\partial_{0}$. Opposed to this, in front-form coordinates the operator is linear in the light-cone time derivative $\partial_{+}$and is always multiplied by a light-cone space derivative $\partial_{-}$.

To illustrate the basic point we follow Robertson [7]. Consider a scalar field in $1+1$ dimensions interacting via a $\phi^{4}$ potential. The Euler-Lagrange equation is $\left(2 \partial_{-} \partial_{+}-m^{2}\right) \phi=$ $-\lambda \phi^{3}$. Compactifying the light-cone space variable $x^{-}$to the finite interval $[-L,+L]$ one can expand the fields in Fourier modes with discrete momenta $k^{+}=\frac{n \pi}{L}, n$ an integer. For $n=0$ there is a zero mode defined by

$$
\langle\phi\rangle_{o} \equiv \int_{+L}^{-L} \frac{d x^{-}}{2 L} \phi(x) .
$$

The equation for this mode is obtained by integrating both sides of the equation of motion over the entire finite interval resulting in

$$
m^{2}\langle\phi\rangle_{o}=\lambda\left(\langle\phi\rangle_{o}\right)^{3}+\lambda \int_{-L}^{+L} \frac{d x^{-}}{2 L}\left(3\langle\phi\rangle_{o}\left(\langle\phi\rangle_{n}\right)^{2}+\left(\langle\phi\rangle_{n}\right)^{3}\right)
$$

where $\langle\phi\rangle_{n}$ is the sum of non-zero Fourier modes. Quite naturally, $\langle\phi\rangle_{o}$ and $\langle\phi\rangle_{n}$ are complementary as $\langle\phi\rangle_{n} \equiv \phi-\langle\phi\rangle_{0}$. So we call $\langle\phi\rangle_{n}$ the 'normal mode' field. The remarkable 
feature of Eq. (3) is that the time derivative has dropped out. The zero mode is thus exposed to be constrained. It is determined by the nonlinear Eq. (3) and not by a dynamical Hamiltonian or Heisenberg equation like $\frac{\partial}{\partial x^{+}}\langle\phi\rangle_{o}=\left\{P^{-},\langle\phi\rangle_{o}\right\}$. One therefore cannot specify independent classical Poisson brackets or corresponding quantum commutators for this mode. This has been known since the 70s with the treatment of Maskawa and Yamawaki [8] via the Dirac constraint method [9]. Indeed, in the Dirac formalism the above constraint appears as one of Second Class, as also seen in ref. [10]. In general, constraints pose a problem in a Hamiltonian formulation to the extent that one should only quantize independent degrees of freedom. Constrained zero modes turn out to be responsible for symmetry breaking in $\phi^{4}$ theory in $1+1$ dimensions $[11,7]$. The work of Bender, Pinsky and van de Sande [12] is particularly lucid on this point. Other theories such as Yukawa and $\phi^{3}$ have also been investigated [13,14]. As a matter of fact, Franke et al. [15] have noticed such a constraint in non-Abelian gauge theory as early as 1981 . However, not even this Leningrad group could overcome the difficulties in the $\mathrm{SU}(2)$ constraint equation.

Here is the problem in $3+1$ dimensional gauge theory [16]: the zero modes proper and the gauge setting are almost inextricably intertwined. Because of these difficulties we complete in the present paper the analysis for the Abelian problem. The lessons learned from this are already being applied to non-Abelian gauge theory [17].

To begin with there is an immediate problem with the choice of gauge traditionally used in light-cone quantization - the light-cone gauge $A^{+}=0$. This is precisely a zero mode problem but, unlike the above, one that is not peculiar to the light-cone but endemic to any compactification of a gauge theory $[18,19]$. Of course one could try omitting the 'torus framework' and remain in the continuum. But then the infrared problem returns to haunt one, for example in the axial gauge propagator which in the literature is a story in its own right $[20]$. We shall therefore persist with the well-defined setting with periodic boundary conditions in all three space directions. Our intention here is thus to first cleanly disentangle the gauge problem from the zero mode problem and to formulate a consistent gauge for handling these modes. This then will enable us to write down the constraint appropriate for quantum electrodynamics as a well defined problem and to elucidate its structure. It remains to be seen whether this is only a formal achievement, or whether it eventually is physically relevant for the long range aspects of quantum chromodynamics. 


\section{Analysis of Maxwell Theory}

Beginning with the standard Maxwell Lagrangian with the photon coupled to an external current, the Euler-Lagrange equations are the usual Maxwell equations

$$
\partial_{\mu} F^{\mu \nu}=J^{\nu} .
$$

For details the reader is referred to the appendix. We consider the theory in a finite volume with $-L \leq x^{-} \leq+L$ and $-L_{\perp} \leq x^{i} \leq+L_{\perp}(i=1,2)$. The gauge fields $A_{\mu}$ and their first derivatives are assigned periodic boundary conditions in each direction of space $\left(x^{-}, x, y\right)$. They are assumed to be smooth in the domain including the boundary. This compactification leads to a discretization in momentum space.

We intend to perform on the Maxwell theory the procedure discussed in the introduction. It is therefore convenient to write the equations (4) explicitly for $\nu=(+,-, i)$

$$
\begin{aligned}
\left(\partial_{-} \partial_{+}-\nabla_{\perp}^{2}\right) A^{+}-\partial_{-}^{2} A^{--}+\partial_{-} \nabla_{\perp} \cdot A_{\perp} & =J^{+}, \\
\left(\partial_{-} \partial_{+}-\nabla_{\perp}^{2}\right) A^{-}-\partial_{+}^{2} A^{+}+\partial_{+} \nabla_{\perp} \cdot A_{\perp} & =J^{-}, \\
\left(2 \partial_{-} \partial_{+}-\nabla_{\perp}^{2}\right) A_{\perp}^{i}+\partial_{i} \partial_{+} A^{+}+\partial_{i} \partial_{-} A^{-}-\partial_{i} \nabla_{\perp} \cdot A_{\perp} & =J^{i} .
\end{aligned}
$$

As in the scalar theory, one integrates these over $x^{-}$and obtains

$$
\begin{aligned}
-\nabla_{\perp}^{2}\left\langle A^{+}\right\rangle_{o} & =\left\langle J^{+}\right\rangle_{o}, \\
-\nabla_{\perp}^{2}\left\langle A^{-}\right\rangle_{o}-\partial_{+}^{2}\left\langle A^{+}\right\rangle_{o}+\partial_{+} \nabla_{\perp} \cdot\left\langle A_{\perp}\right\rangle_{0} & =\left\langle J^{-}\right\rangle_{o}, \\
-\nabla_{\perp}^{2}\left\langle A_{\perp}^{i}\right\rangle_{0}+\partial_{i} \partial_{+}\left\langle A^{+}\right\rangle_{o}-\partial_{i} \nabla_{\perp} \cdot\left\langle A_{\perp}\right\rangle_{0} & =\left\langle J^{i}\right\rangle_{o},
\end{aligned}
$$

with the zero mode defined in the same way as in Eq. (2). Subtracting the latter equations from the former gives the normal mode equations

$$
\begin{aligned}
\left(\partial_{-} \partial_{+}-\nabla_{\perp}^{2}\right)\left\langle A^{+}\right\rangle_{n}-\partial_{-}^{2}\left\langle A^{-}\right\rangle_{n}+\partial_{-} \nabla_{\perp} \cdot\left\langle A_{\perp}\right\rangle_{n} & =\left\langle J^{+}\right\rangle_{n} \\
\left(\partial_{-} \partial_{+}-\nabla_{\perp}^{2}\right)\left\langle A^{-}\right\rangle_{n}-\partial_{+}^{2}\left\langle A^{+}\right\rangle_{n}+\partial_{+} \nabla_{\perp} \cdot\left\langle A_{\perp}\right\rangle_{n} & =\left\langle J^{-}\right\rangle_{n} \\
\left(2 \partial_{-} \partial_{+}-\nabla_{\perp}^{2}\right)\left\langle A^{i}\right\rangle_{n}+\partial_{i} \partial_{+}\left\langle A^{+}\right\rangle_{n}+\partial_{i} \partial_{-}\left\langle A^{-}\right\rangle_{n}-\partial_{i} \nabla_{\perp} \cdot\left\langle A_{\perp}\right\rangle_{n} & =\left\langle J^{i}\right\rangle_{n}
\end{aligned}
$$

Note that the zero and normal mode equations decouple into two independent sectors. The latter sector has been the basis for previous DLCQ treatments of QED $[4,5,6]$.

Now we shall set the gauge using the symmetry $A_{\mu} \rightarrow A_{\mu}-\partial_{\mu} \omega$. Of course, one may choose different gauges for the zero and normal modes, $\left\langle A^{\mu}\right\rangle_{o}$ and $\left\langle A^{\mu}\right\rangle_{n}$, respectively. This holds true even in $\mathrm{SU}(\mathrm{N})$ where the sectors are not as nicely decoupled as above. Naturally, isolating zero modes is senseless if boundary conditions can change under gauge 
transformations. Therefore $\partial_{\mu} \omega$ must also be periodic. As a matter of fact, $\omega$ itself must be periodic for it to be a topologically trivial element of $U(1)$. Standard gauge fixing is done precisely in this sector of the gauge group ${ }^{1}$. One sees then that the zero mode $\left\langle A^{+}\right\rangle_{0}$ is invariant under the allowable gauge transformations; it cannot be 'gauged away'.

Gauge Setting for Normal Modes. By the above considerations we are compelled to conclude that the traditional light-cone gauge $A^{+}=0$ cannot be used for all modes. But a modified light-cone gauge is admissible, namely

$$
\left\langle A^{+}\right\rangle_{n}=0
$$

It is the unique solution to $\partial_{-} A^{+}=0$ and is consistent with practically all preceding work on DLCQ $[4,5,6]$. These works are restricted to the normal mode sector without the authors being explicit about it. Note that this gauge choice still admits $x^{--}$-independent gauge transformations to be discussed below.

The above gauge renders Eq.(11) invertible: the time derivative drops out and the equation becomes a constraint for $\left\langle A^{-}\right\rangle_{n}$. The inversion of $\left(\partial_{-}\right)^{2}$ becomes a well-defined operation since there are no zero modes on the right hand side. Having eliminated $\left\langle A^{-}\right\rangle_{n}$ as an independent field one can proceed with writing the full Hamiltonian purely in terms of dynamical quantities $\left\langle A^{i}\right\rangle_{n}$ (and the sources $J^{\mu}$ ). With dynamical fermions included one will obtain the Hamiltonian in [4], the basis for the work of $[5,6]$. This completes our considerations in the normal mode sector. We now specifically pursue the zero modes.

Simple, Global and Proper Zero Modes. The zero modes, of course, are still functions of $x_{\perp}$. Thus here we are again: Eq.(8), $\nabla_{\perp}^{2}\left\langle A^{+}\right\rangle_{0}=\left\langle J^{+}\right\rangle_{o}$, is a constraint for $\left\langle A^{+}\right\rangle_{o}$, but before one can invert it one must remove 'zero modes' from the right hand side. We therefore introduce for some field $\phi$ the space average

$$
\langle\phi\rangle \equiv \int \frac{d^{3} x}{\Omega} \phi(x)
$$

which we refer to as the global zero mode as opposed to the simple zero mode $\langle\phi\rangle_{o}$ used above. The difference of these two quantities

$$
\stackrel{\circ}{\phi} \equiv\langle\phi\rangle_{o}-\langle\phi\rangle
$$

\footnotetext{
${ }^{1}$ One must be able to ignore surface terms. In a Hamiltonian formulation, the Gauss law operator denoted, say, by $G$ is the generator of infinitesmal gauge transformations on the spatial gauge field components via $\delta A_{\alpha}(x)=\int d y \omega(y)\left\{A_{\alpha}(x), G(y)\right\}$. Here $\alpha=-, i$ and $\{$,$\} is the Poisson bracket. The result$ is $\partial_{\alpha} \omega(x)$ only if one can discard the surface terms of $\omega$. Gauge functions $\omega$ with nonperiodic boundary conditions give homotopically nontrivial gauge transformations which are not unimportant [19]. But we postpone these for future investigation.
} 
will be referred to as the proper zero mode. By analogy with the above, we integrate Eqs.(8)-(10) over $\vec{x}_{\perp}$ to arrive at the 'equations of motion' in the global zero mode sector

$$
\begin{aligned}
0 & =\left\langle J^{+}\right\rangle, \\
-\partial_{+}^{2}\left\langle A^{+}\right\rangle & =\left\langle J^{-}\right\rangle, \\
0 & =\left\langle J^{i}\right\rangle .
\end{aligned}
$$

Subtracting these from Eqs.(8)-(10) gives the 'equations of motion' in the proper zero mode sector

$$
\begin{aligned}
& -\nabla_{\perp}^{2} \stackrel{o}{A^{+}}=\stackrel{o}{J^{+}} \\
& -\partial_{+}^{2} \stackrel{o}{A^{+}}-\nabla_{\perp}^{2} \stackrel{o}{A^{-}}+\partial_{+} \nabla_{\perp} \cdot \stackrel{\circ}{A_{\perp}}=\stackrel{o}{J^{-}} \text {, } \\
& -\nabla_{\perp}^{2} \stackrel{o}{A^{i}}+\partial_{i} \partial_{+} \stackrel{o}{A^{+}}-\partial_{i} \nabla_{\perp} \cdot \stackrel{o}{A_{\perp}}=\stackrel{o}{J^{i}} \text {. }
\end{aligned}
$$

We have now completely separated all possible zero modes corresponding to the three space directions. The equations of motion for each sector contain no ambiguities.

Gauge Setting for Zero Modes. The first of Eqs.(17)-(19) is the Gauss law in the global sector. It expresses that, given the boundary conditions on the fields, the net charge in the theory must be zero. In the quantum theory this is implemented as a condition on physical state vectors of the theory, not as an operator identity. In other words the quantum theory we eventually build is only valid between charge singlet states. For a study of positronium $[4,5,6]$ this of course is not restrictive. With Eq.(17) satisfied in this way, Eq.(19) is satisfied simultaneously.

The second of these equations, Eq.(18), expresses that the global zero mode of $A^{+}$is a dynamical degree of freedom. In the quantum theory it therefore leads to quanta with the same quantum numbers as the vacuum. This mode has been known in the past in other guises. In the Schwinger model its analogue is known to lead to multiple vacua $[19,21,22]$. Its presence in $3+1$ dimensions was noticed in [23]. Since the longitudinal electric field is given by $E_{\|}=F_{+-}$, the global mode $\left\langle A^{+}\right\rangle$describes a purely time dependent background field $\left\langle E_{\|}\right\rangle=\partial_{+}\left\langle A^{+}\right\rangle$. But according to $[21]$, it should not play any role since it is energetically favourable for the 'vacuum' to generate pairs until the background electric field is eliminated. One would, however, expect such a field to play a significant role in QCD.

The first of the second set, Eqs.(20)-(22), now is a soluble equation. The Green's function corresponding to the inversion of $\nabla_{\perp}^{2}$ is given in the appendix. However, without gauge setting the remaining two equations of this set are ambiguous. A natural gauge 
choice in this sector is

$$
\nabla_{\perp} \cdot \stackrel{o}{A_{\perp}}=0
$$

The gauge function $\stackrel{o}{\omega}$ which brings an arbitrary configuration $A_{\mu}^{\prime}$ to this gauge satisfies $\nabla_{\perp}^{2} \stackrel{o}{\omega}=\nabla_{\perp} \cdot \stackrel{o}{A_{\perp}^{\prime}}$. It is $x^{-}$-independent and therefore does not disturb the previous gauge condition. Now there is no freedom left to perform spatially dependent gauge transformations. With this gauge and trivial manipulations the above equations generate the constraints determining $\stackrel{\circ}{A^{+}}, \partial_{+} \stackrel{\circ}{A^{+}}$, and $\stackrel{\circ}{A^{i}}$, namely

$$
\begin{aligned}
& \stackrel{\circ}{A^{+}}=-\frac{1}{\nabla_{\perp}^{2}} \stackrel{\circ}{J^{+}} \quad, \quad \partial_{+} \stackrel{\circ}{A^{+}}=\frac{1}{\nabla_{\perp}^{2}} \partial_{i} \stackrel{o}{J^{i}}, \\
& \stackrel{\circ}{A^{i}}=-\frac{1}{\nabla_{\perp}^{2}}\left(g^{i j}+\frac{\partial^{i} \partial^{j}}{\nabla_{\perp}^{2}}\right) \stackrel{o}{J_{j}} .
\end{aligned}
$$

As for $\stackrel{\circ}{A^{-}}$, Eq. (21) should be considered its definition. The latter cannot be a dynamical equation for $A^{+}$as one might think at first, because ${ }^{+}$including all its time derivatives is given by the first of Eqs.(24). However, $\stackrel{\circ}{A}^{-}$is simply not needed for the Hamiltonian. It appears in $P^{-}$only in the combination $\stackrel{0}{A^{-}}\left(\nabla_{\perp}^{2} \stackrel{o}{A^{+}}+\stackrel{o}{J^{+}}\right)$which obviously vanishes. We therefore do not give it explicitly.

Three global zero modes have not appeared at all yet, namely $\left\langle A^{-}\right\rangle$and $\left\langle A^{i}\right\rangle$. One of them can be removed by a further gauge choice

$$
\left\langle A^{-}\right\rangle=0
$$

The gauge function $\langle\omega\rangle$ bringing the arbitrary configuration $A_{\mu}^{\prime}$ to this gauge satisfies $\partial_{+}\langle\omega\rangle=\left\langle A_{+}^{\prime}\right\rangle$. Since it depends only on the light-cone time it is not in conflict with any of the above gauge or boundary conditions. With the latter condition, all gauge freedom is exhausted. The global zero mode of the transverse fields therefore remain unspecified. Neither a constraint nor dynamical equations of motion determine them. At the level of the Maxwell theory they would appear to be unnecessary quantities but we shall see in the next section that the solution of the constrained fermion component reintroduces them into the field equations. This has been also pointed out by McCartor ${ }^{2}$ and we comment on it later, again.

A final remark is in order. By the gauge condition, Eq. (26), one has removed $\left\langle A^{-}\right\rangle$as an independent degree of freedom. The Euler-Lagrange equation involving the variation $\delta \mathcal{L} / \delta\left(\left\langle A^{-}\right\rangle\right)$can no longer occur. But this is precisely the global Gauss law, Eq. (17),

\footnotetext{
${ }^{2} \mathrm{McCartor}$ in [23] suggests that $\left\langle A^{i}\right\rangle$ may need to be initialized on the light-cone surface of constant $\boldsymbol{x}^{-}$such as used in [24].
} 
containing physical information. The constraint must therefore be reintroduced in the quantum theory as an explicit condition on physical states as we said above.

Summarizing, the gauge conditions we have implemented are

$$
\left\langle A^{+}\right\rangle_{n}=0, \quad \partial_{i} \stackrel{o}{A^{i}}=0, \quad\left\langle A^{-}\right\rangle=0 .
$$

For lack of a better name, we call the simultaneous imposition of these conditions the Compactification Gauge. In the 'compact' gauge the quantities $\left\langle A^{-}\right\rangle_{n}, \stackrel{o}{A^{-}}, \stackrel{o}{A^{+}}, \partial_{+} \stackrel{o}{A^{+}}$ and ${ }^{i}$ are constrained and given by Eqs.(11), (21), (24) and (25), respectively. The $\left\langle A_{\perp}\right\rangle$ 's are thus far undetermined. The only dynamical quantities are $\left\langle A^{i}\right\rangle_{n}$ and $\left\langle A^{+}\right\rangle$. In other words these are the only three fields for which one is permitted to specify independent commutators in quantizing the theory. The only 'weak' constraint is the vanishing of the net charge $\left\langle J^{+}\right\rangle$. This means we can build a consistent quantum theory only on charge singlet states.

Actually, Robertson [25] has noticed that the compact gauge is a special case of a general gauge fixing involving a parameter $\alpha$ analogous to that in a general covariant gauge.

\section{Structure in the Instant Form}

We now briefly analyze the analogous set up of the theory in the instant form with periodic boundary conditions, where in particular $-L \leq x^{3} \leq+L$. The gauge conditions analogous to the compact gauge in this context have been already introduced in a somewhat different setting by Lenz et al. [26], building on a gauge used by Yabuki [27], that is

$$
\partial_{3} A^{3}=0, \nabla_{\perp} \cdot \stackrel{\circ}{A_{\perp}}=0,
$$

with the zero mode now defined over $x^{3}$. The first condition was also used by Manton [19] for $1+1$ dimensions. To these we could add, again, the condition $\left\langle A^{0}\right\rangle=0$. In the two subsectors of interest the Maxwell equations are

$$
\begin{aligned}
0 & =\left\langle J^{0}\right\rangle, \\
\partial_{0}^{2}\left\langle A^{i}\right\rangle & =\left\langle J^{i}\right\rangle, \\
\partial_{0}^{2}\left\langle A^{3}\right\rangle & =\left\langle J^{3}\right\rangle,
\end{aligned}
$$

in the global zero mode sector, and correspondingly in the proper zero mode sector

$$
-\nabla_{\perp}^{2} \stackrel{o}{A^{0}}+\partial_{0} \nabla_{\perp} \cdot \stackrel{o}{A_{\perp}}=\stackrel{o}{J^{0}}
$$




$$
\begin{aligned}
\left(\partial_{0}^{2}-\nabla_{\perp}^{2}\right) \stackrel{o}{A^{i}}+\partial_{i}\left(\partial_{0} \stackrel{o}{A^{0}}-\nabla_{\perp} \cdot \stackrel{o}{A_{\perp}}\right) & =\stackrel{o}{J^{i}} \\
\left(\partial_{0}^{2}-\nabla_{\perp}^{2}\right) \stackrel{o}{A^{3}} & =J^{3}
\end{aligned}
$$

Obviously, we have separated out the three-direction and retained $i=1,2$. We see that, apart from $A^{0}$ which assumes its usual role as a multiplier of the Gauss law, these degrees of freedom remain dynamical. In a Hamiltonian framework they must have independent Poisson brackets or corresponding quantum commutators. Thus from the point of view of zero modes, equal usual-time and equal light-cone-time quantization are completely different.

\section{Quantum Electrodynamics}

In principle, one could repeat the same considerations for the Dirac equations but now regarding the vector potentials $A^{\mu}$ as given sources for all times. However, we shall be using antiperiodic boundary conditions on the spinor fields, justified by their bilinear appearance in the action. Since they then have no zero modes, this procedure would generate no new insight. Only if one considers the coupled Dirac-Maxwell equations, that is only if one considers full quantum electrodynamics, do some new feedbacks occur, to be discussed in the sequel. We shall see that the constraints for the transverse photon proper zero modes become significantly non-trivial due to the coupled nature of the problem. To avoid the inevitable questions on operator ordering we propose, and follow below, formulating the constraint at the classical level. Operator ordering is left to a future step.

The Problem. Returning then to the constraints given by Eqs.(24) and (25), we observe the obvious feature that in full quantum electrodynamics the sources $J^{\mu}$ are not given for all times but are part of the dynamical problem. In terms of projected spinor components, see the appendix, the currents decompose into

$$
J^{+}=\sqrt{2} g \psi_{+}^{\dagger} \psi_{+}, \quad J^{-}=\sqrt{2} g \psi_{-}^{\dagger} \psi_{-}, \quad \text { and } \quad J^{i}=g\left(\psi_{+}^{\dagger} \alpha^{i} \psi_{-}+\text {h.c. }\right) .
$$

We see that the proper zero mode $\stackrel{\circ}{A^{+}}$only depends on $\psi_{+}$via $J^{+}$, while $\stackrel{0}{A^{i}}$ depends on both $\psi_{+}$and $\psi_{-}$. The latter are coupled by the Dirac equations, thus by

$$
\begin{aligned}
& i D_{+} \psi_{+}=-\frac{1}{\sqrt{2}}\left(i \alpha^{j} D_{j}-m \beta\right) \psi_{-}, \\
& i D_{-} \psi_{-}=-\frac{1}{\sqrt{2}}\left(i \alpha^{j} D_{j}-m \beta\right) \psi_{+} .
\end{aligned}
$$

The first equation contains time derivatives and is dynamical. In a Hamiltonian approach one deals with this by giving initial 'data' for $\psi_{+}$at the surface $x^{+}=0$. Thus $\stackrel{o}{+}^{+}$is 
also known at $x^{+}=0$. Eq.(37) however contains no time derivatives and is a constraint. According to tribal custom, one considers it as the (constraint) equation for $\psi_{-}$. But what must be given to determine it at the initial surface $x^{+}=0$ unambiguously? In passing, we mention that a consideration of bosonic zero modes in heavy fermion theories quantized on the light-cone has been carried out by Brown et al. [28]. There the heavy mass limit causes the fermions $\psi_{+}$and $\psi_{-}$to decouple removing the fermion constraint. Thus the following constraints do not arise in their considerations.

The Notation. Imagine that one has succeeded in inverting the derivative $D_{-}=\partial_{-}+$ $i g\left\langle A^{+}\right\rangle_{o}$. There is nothing ill-defined about this inversion. One actually can write down a Green's function which safely allows this but we do not quote it here. Then $\psi_{-}$stands alone on the left hand side of Eq.(37). On the right hand side, one has ${ }^{+}$(which we now know at $\left.x^{+}=0\right)$, the dynamical global zero mode $\left\langle A^{+}\right\rangle$, the dynamical fermion field $\psi_{+}$, the dynamical photons $\left\langle A^{i}\right\rangle_{n}$, and finally the proper zero modes $\stackrel{\circ}{*}^{i}$. Here is where the snake bites its tail: to determine $\stackrel{o}{A}^{i}$ we need $\psi_{-}$for which we need the very same $\stackrel{o}{A}^{i}$. The procedure is now to implement the constraint Eq.(37), insert it into the current $J^{i}$ and thus write the complete constraint arising from the Maxwell constraint, Eq.(25). Obviously, things are very complicated and we need some appropriate notation to disentangle the essential features. We thus introduce $S, R_{i}$, and $U_{i}$ which are functionals of $D_{-}, \psi_{+}$, and $\left\langle A^{i}\right\rangle_{n}$ in the manner indicated by

$$
\begin{aligned}
S\left[D_{-}, \psi_{+}\right] & \equiv \psi_{+}^{\dagger} \frac{1}{i D_{-}} \psi_{+}, \\
R_{i}\left[D_{-}, \psi_{+}\right] & \equiv \psi_{+}^{\dagger} \alpha_{i} \frac{1}{i D_{-}} \tilde{\psi}_{-}+\text {h.c. } \\
U_{i}\left[D_{--}, \psi_{+},\left\langle A_{\perp}\right\rangle_{n}\right] & \equiv \psi_{+}^{\dagger} \alpha_{i} \alpha_{j} \frac{1}{i D_{-}}\left(\left\langle A^{j}\right\rangle_{n} \psi_{+}\right)+\text {h.c. }
\end{aligned}
$$

We have also used

$$
\begin{aligned}
\tilde{\psi}_{-} & =\left(i \alpha_{j} \partial^{j}-m \beta\right) \psi_{+} \\
T^{i j} & =-\frac{1}{\nabla_{\perp}^{2}}\left(g^{i j}+\frac{\partial^{i} \partial^{j}}{\nabla_{\perp}^{2}}\right)
\end{aligned}
$$

as abbreviations. Note that $\tilde{\psi}_{-}$is the free field solution to the $\psi_{-}$Dirac equation [2].

The Equation. Following the procedure discussed directly above one arrives at

$$
\stackrel{\circ}{A^{i}}+\sqrt{2} g^{2} T^{i j}\left(\stackrel{\circ}{S} \stackrel{o}{A}_{j}\right)=-\frac{1}{\sqrt{2}} g\left[T^{i j}\left(\stackrel{\circ}{R_{j}}\right)-g T^{i j}\left(\stackrel{o}{U_{j}}\right)+2 g\left\langle A_{j}\right\rangle T^{i j}(\stackrel{o}{S})\right] .
$$

Of course $T^{i j}$, being an integro-differential operator, acts on all quantities to the right enclosed by the parentheses $(\cdot)$. Obviously, the notation was adapted such that the unknown 
proper zero mode $\stackrel{o}{i}^{i}$ appears only on the left hand side. Since the equation is linear in the $A^{i}$, one can invert it provided the right hand side is given. Obviously, the latter depends on $\left\langle A^{i}\right\rangle_{n},\left\langle A^{+}\right\rangle$and $\psi_{+}$. These are given, since they are specified on the initial surface. But the global zero mode $\left\langle A^{i}\right\rangle$, sneaking through the back door, shows up in the last term on the right hand side. It must be given before one can invert the equations. One possibility is mentioned below.

An Alternate Equation. Before continuing one should mention that the appearance of $D_{-}$ perhaps needlessly overcomplicates the form of the constraint Eq.(43). We now consider an alternative formulation, where we remove $D_{-}$by taking new variables

$$
\varphi_{ \pm}=e^{i g x^{-}\left\langle A^{+}\right\rangle_{o}} \psi_{ \pm}
$$

and instead of imposing antiperiodic boundary conditions on the $\psi$, introduce them on the new spinor fields $\varphi$. The Dirac equations change to

$$
\begin{aligned}
i\left(D_{+}-i g x^{-}\left\langle\pi^{-}\right\rangle_{0}\right) \varphi_{+} & =-\frac{1}{\sqrt{2}}\left(i \alpha^{j} D_{j}-m \beta+g x^{-} \alpha^{j}\left\langle\pi_{j}\right\rangle_{o}\right) \varphi_{-}, \\
i \partial_{-} \varphi_{-} & =-\frac{1}{\sqrt{2}}\left(i \alpha^{j} D_{j}-m \beta+g x^{-} \alpha^{j}\left\langle\pi_{j}\right\rangle_{o}\right) \varphi_{+} .
\end{aligned}
$$

We have written these in terms of $\left\langle\pi_{j}\right\rangle_{o}=\partial_{j}\left\langle A^{+}\right\rangle_{o}=\partial_{j} \stackrel{o}{A^{+}}$, which is nothing but the conjugate momentum of the photon field $A^{i}$. Observe that the space derivative kills the spatially constant $\left\langle A^{+}\right\rangle$. Thus, at least in this formulation, the dynamical mode $\left\langle A^{+}\right\rangle$ drops out of the constraint. The currents $J^{\mu}$ have the same form as above but with $\psi$ replaced by $\varphi$. We can use the same notation as in the previous case by taking $S, R_{i}$ and $U_{i}$ as functionals of the ordinary derivative $\partial_{-}$and $\varphi_{+}$. Thus instead of the constraint (43), one could consider

$$
\stackrel{o}{A^{i}}+\sqrt{2} g^{2} T^{i j}\left(\stackrel{o}{S} A_{j}\right)=-\frac{1}{\sqrt{2}} g\left[T^{i j}\left(\stackrel{o}{R}_{j}\right)-g T^{i j}\left(\stackrel{o}{U_{j}}\right)-\sqrt{2} g^{2} T^{i j}\left(\stackrel{o}{V_{j}}\right)+2 g\left\langle A_{j}\right\rangle T^{i j}(\stackrel{o}{S})\right],
$$

where

$$
V_{i}\left[\partial_{-}, \varphi_{+}\right] \equiv \varphi_{+}^{\dagger} \alpha_{i} \alpha_{j} \frac{\partial^{j}}{\nabla_{\perp}^{2}}(\stackrel{o}{\rho}) \frac{1}{i \partial_{-}}\left(x^{-} \varphi_{+}\right)+\text {h.c. }, \rho \equiv \varphi_{+}^{\dagger} \varphi_{+}
$$

absorbs the new term in this alternative formulation. Eqs.(43) and (47) are the central results of this paper.

\section{Solving the Constraint Iteratively}

Let us first regard that somehow the global zero modes $\left\langle A^{i}\right\rangle$ can be specified at the initial surface $x^{+}=0$. Standard (Fredholm) techniques can then be applied to solve Eq.(43) 
and Eq.(47): one takes the Fourier transform using the convolution theorem and iterates to deal with the convolution term. As is already evident in the structure of Eq.(43) this generates precisely an expansion in the coupling constant $g$, though there are of course factors of $g$ buried in $D_{-}$. The weak coupling nature of the iterative solution is more evident using the alternate formulation Eq.(47). To be slightly more specific let us write this out for the case of the alternate formulation, at least schematically. We introduce the lower case quantities $a^{i}, s$ and $t^{i j}$ to denote the corresponding Fourier transform of the objects $\stackrel{\circ}{A^{i}}, \stackrel{o}{S}$ and $T^{i j}$. We summarise the Fourier transforms of the inhomogeneous terms by $g \omega_{(1)}^{i}+g^{2} \omega_{(2)}^{i}+g^{3} \omega_{(3)}^{i}$ and absorb factors like $\sqrt{2}$ into the definitions. Then the constraint Eq.(47) is in momentum space,

$$
a^{i}\left(p_{\perp}\right)+g^{2} \sum_{q_{\perp} \neq 0} t^{i j}\left(p_{\perp}\right) s\left(p_{\perp}-q_{\perp}\right) a_{j}\left(q_{\perp}\right)=g \omega_{(1)}^{i}\left(p_{\perp}\right)+g^{2} \omega_{(2)}^{i}\left(p_{\perp}\right)+g^{3} \omega_{(3)}^{i}\left(p_{\perp}\right) .
$$

Recall that the space compactification discretizes the momenta. After some reorganisation, the solution after the second iteration clearly shows the power series in the coupling constant $g$ that develops

$$
\begin{aligned}
a_{(2)}^{i}\left(p_{\perp}\right) & =g \omega_{(1)}^{i}\left(p_{\perp}\right)+g^{2} \omega_{(2)}^{i}\left(p_{\perp}\right)+g^{3} \omega_{(3)}^{i}\left(p_{\perp}\right) \\
& -g^{3} \sum_{q_{\perp} \neq 0} t^{i j}\left(p_{\perp}\right) s\left(p_{\perp}-q_{\perp}\right) \omega_{j(2)}\left(q_{\perp}\right) \\
& -g^{4} \sum_{q_{\perp} \neq 0} t^{i j}\left(p_{\perp}\right) s\left(p_{\perp}-q_{\perp}\right) \omega_{j(3)}\left(q_{\perp}\right) .
\end{aligned}
$$

where we have arranged the terms in order of ascending powers of $g$.

This method of solution is clearly forcing one to study the contributions such terms make in perturbation theory although the order of iteration is not matched exactly with the order in $g$. Note that the zero mode for which we are solving appears in the canonical Hamiltonian $P^{-}$quadratically. Thus the lowest order contributions to the Hamiltonian in perturbation theory come from just the order $g$ term in this solution, namely $\omega_{(1)}^{i}$ which only involves the free field dynamical fermion $\varphi_{+}$. Only at second order need one consider the dynamical photons $\left\langle A^{i}\right\rangle_{n}$, the global zero mode (if supposed to be a dynamical variable), and any potential differences between the two formulations of the fermions presented earlier.

Here we have studied only the constraint. A detailed study of its impact on the Hamiltonian $P^{-}$is still in progress [29]. We can nevertheless mention that to this order at least, the dynamical global mode $\left\langle A^{+}\right\rangle$does not play a role. This is a consequence of the charge singlet formulation of the quantum theory taken together with the structure of the 
interaction term for this field, namely $\left\langle A^{+}\right\rangle\left\langle J^{-}\right\rangle$. The current in this expression, to this order, is just proportional to the charge $\left\langle J^{+}\right\rangle$and so transitions between different 'vacua' cannot be generated when applied to the physical charge singlet states. This is precisely the 'Coleman mechanism': "one could introduce a constant background electric field, but it wouldn't matter" [21] - at least to first order in perturbation theory.

Treatment of $\left\langle A^{i}\right\rangle$. Finally, we propose a method for determining the space-independent modes $\left\langle A^{i}\right\rangle$. In the above solution (50) they sit in the term $\omega_{(2)}^{i}$. These modes remind one of the standard infrared problem of quantum electrodynamics - very soft photons. In our way of formulating quantum electrodynamics they are truly zero momentum photons. The usual treatment is to give the photon a small mass, while not worrying over violations of gauge invariance and residual effects in the mass-to-zero limit because one simply cannot measure infinitely soft photons with a finite resolution apparatus [30]. We now show how a similar treatment for the global zero mode $\left\langle A^{i}\right\rangle$ allows it to be determined, and sketch how it turns out to be irrelevant to lowest order. We assign a small but finite mass only to the global transverse zero modes by adding the term $\mu^{2}\left\langle A_{\perp}\right\rangle \cdot\left\langle A_{\perp}\right\rangle$ to the Lagrangian. The equation of motion corresponding to this field then is, instead of Eq.(19),

$$
\left\langle A^{i}\right\rangle=\frac{1}{\mu^{2}}\left\langle J^{i}\right\rangle .
$$

Putting in the currents and following the procedure that lead to Eq.(43) or Eq.(47) gives a constraint for the global zero modes $\left\langle A^{i}\right\rangle$. This constraint is similar in structure to the proper zero mode constraints, Eq.(43) or Eq.(47), but with $T^{i j}$ replaced by just the mass $\mu^{2}$ on the left hand side. On the right hand side of this equation are all the dynamical fields as well as the proper zero mode $\stackrel{o}{A}^{i}$. The lowest order iterative solution to the global constraint then is already of order $g$. When fed back into Eq.(43) or Eq.(47), the $\mu$ dependent terms will be of order $g^{3}$. Therefore they are absorbed into $\omega_{(3)}^{i}$ in the solution Eq.(50) and drop out to lowest order.

\section{Summary and Conclusions}

In this paper we have given a careful analysis of quantum electrodynamics in a space of periodic gauge potentials $A^{\mu}$ and antiperiodic fermion fields $\psi$ in the framework of front form field theory. We have determined which gauge conditions can be set in general and, in such a gauge, how the Maxwell equations and combined Dirac-Maxwell equations can be solved consistently and unambiguously. In particular, with the compactification gauge, the dynamical fields are the normal mode potentials $\left\langle A^{i}\right\rangle_{n}$, the global zero mode $\left\langle A^{+}\right\rangle$ 
and the spinor component $\psi_{+}$. The notation and nomenclature were developed in Sect.2. There are also constrained fields. These are the fermion component $\psi_{-}$, the proper zero mode ${ }^{\circ}+$ and most significantly the proper zero modes of the transverse gauge potentials $\stackrel{\circ}{A^{i}}$. The constraint equations for these fields were found to be rather nontrivial. We obtained their solutions by iteration which was seen to be a perturbation expansion in weak coupling.

The major conclusion to be drawn is that we now have a method by which to tackle the problem of identifying the role of the various zero mode fields in a gauge theory: namely to project out the zero modes and construct compatible gauge conditions permitting either identification of the field as dynamical or constrained. This is a new insight. We expect it will enable us to make progress on the problems which halted earlier attempts at understanding the zero modes for quantum chromodynamics.

To some extent disappointingly, we have not succeeded in solving the constraints nonperturbatively, in three space dimensions at least. On the other hand, apart from speculations that there may exist a strong coupling phase of QED, the results of such a solution here are purely academic. The comparable constraint in QCD will be vastly different due to the non-Abelian coupling, as we have learned from continuing work underway [17]. As well, a careful study of the contributions the constrained modes make in the weak coupling Hamiltonian of QED is in progress [29]. 


\section{Acknowledgements}

The authors are indebted to discussions with Professors Steve Pinsky and James Vary, Drs. Ľubomír Martinovič, Dave Robertson, Pavel Gornicki and Rik Naus, as well as with Stefan Güllenstern and Stephan Elser. ACK is supported by the DFG under contract DFG-Gz:Pa 450/1-1.

\section{Appendix: Notation and Conventions}

Light-Cone Coordinates. The convention for light-cone coordinates we employ is that of Kogut and Soper [31]: $x^{ \pm}=\left(x^{0} \pm x^{3}\right) / \sqrt{2}$ and $\left(x^{1}, x^{2}\right)$ unchanged. The four-vector dot product decomposes as $A \cdot B=A^{+} B^{-}+A^{-} B^{+}-A^{i} B^{i}$ where latin indices $(i, j, k \ldots=1,2)$ are over the transverse components and we shall use $A_{\perp} \cdot B_{\perp} \equiv A^{i} B^{i}$. Following Dirac $[1], x^{+}$is taken as the time parameter. The time derivative is thus $\partial_{+} \equiv \partial / \partial x^{+}$and the light-cone metric tensor $g^{\mu \nu}$ leads to $\partial_{+}=\partial^{-}$. Correspondingly, $\partial_{-}=\partial / \partial x^{-}=\partial^{+}$is a space derivative.

Quantum Electrodynamics. The Lagrangian density of Quantum Electrodynamics is given by

$$
\mathcal{L}=-\frac{1}{4} F^{\mu \nu} F_{\mu \nu}+\bar{\psi}(i \not D-m) \psi
$$

with the standard definitions of the field strength tensor, covariant derivative and 'slash' notation namely

$$
F^{\mu \nu}=\partial^{\mu} A^{\nu}-\partial^{\nu} A^{\mu}, \quad D_{\mu}=\partial_{\mu}+i g A_{\mu}, \quad \text { and } \quad \not D=\gamma^{\mu} D_{\mu} .
$$

The spinor $\bar{\psi}$ means $\psi^{\dagger} \gamma_{0}$ and we use the Dirac notation $\alpha^{i}=\gamma^{0} \gamma^{i}, \beta=\gamma^{0}$. The spinors $\psi_{ \pm}$are given by $\psi_{ \pm}=\Lambda^{ \pm} \psi$ where $\Lambda^{ \pm} \equiv \frac{1}{\sqrt{2}} \gamma^{0} \gamma^{ \pm}$and $\gamma^{ \pm} \equiv\left(\gamma^{0} \pm \gamma^{3}\right) / \sqrt{2}$. If not otherwise stated we work in the chiral representation where $\psi_{+}$has entries only in positions 1 and 4 of the four-spinor and $\psi_{-}$is non-zero only in positions 2 and 3.

Delta functions. The definition of the delta function on a space of periodic functions was given in the appendix of our earlier work [16]. Here we define

$$
\begin{aligned}
& \delta_{p}(x)=\frac{1}{2 L}+\frac{1}{2 L} \sum_{n=-\infty}^{\infty} \exp (i \pi n x / L), \\
& \delta_{a}(x)=\frac{1}{2 L} \sum_{n= \pm \frac{1}{2}, \ldots} \exp (i \pi n x /(L)) .
\end{aligned}
$$

where we have changed notation slightly: ' $p$ ' signifies the validity for periodic functions. The hash through the summation symbol denotes the exclusion of $n=0$ in the sum. The 
two pieces of that, the zero mode and normal mode parts, satisfy completeness respectively in the spaces of zero mode and normal mode functions. The ' $a$ ' indicates validity for antiperiodic functions.

Green's Functions. The Green's functions related to the inversions of $\partial_{-}$and $\partial_{-}^{2}$ acting on periodic functions are

$$
\begin{aligned}
\mathcal{E}_{L}^{(p)} & =\frac{1}{2 i \pi} \sum_{n=-\infty}^{\infty} \frac{1}{n} \exp (i \pi n x / L), \\
\zeta_{L}^{(p)}(x) & =-\frac{L}{2 \pi^{2}} \sum_{n=-\infty}^{\infty} \frac{1}{n^{2}} \exp (i \pi n x / L),
\end{aligned}
$$

respectively. The Green's function related to the two dimensional Laplacian acting on periodic functions is

$$
\eta_{L}^{(p)}\left(x_{\perp}\right)=-\frac{L}{2 \pi^{2}} \sum_{n, m}^{\prime} \frac{1}{n^{2}+m^{2}} \exp \left(i \pi\left(n x^{1}+m x^{2}\right) / L\right)
$$

where the $\Sigma^{\prime}$ indicates that the single point $n=m=0$ is excluded in the double summation, though $n$ and $m$ can be individually zero. Finally,

$$
\mathcal{E}_{L}^{(a)}=\frac{1}{i \pi} \sum_{n= \pm \frac{1}{2}, \ldots} \frac{1}{n} \exp (i \pi n x / L)
$$

is the Green's function for inverting $\partial_{-}$acting on an antiperiodic functions. 


\section{References}

[1] P.A.M. Dirac: Rev. Mod. Phys. 21 (1949) 392; H. Leutwyler, J. Stern: Ann. Phys. (N.Y.) 112 (1978) 94.

[2] S.J. Brodsky, H.C. Pauli: Light-Cone Quantization of Quantum Chromodynamics in Recent Aspects of Quantum Fields, eds. H. Mitter and H. Gausterer, Lecture Notes in Physics, Vol. 396. Berlin, Heidelberg, New York: Springer-Verlag 1991.

[3] H.C. Pauli, S.J. Brodsky: Phys. Rev. D32 (1985) 1993; (1985) 2001; T. Eller, H.C. Pauli, S.J. Brodsky: Phys. Rev. D35 (1987) 1493; K. Hornbostel, S.J. Brodsky, H.C. Pauli: Phys. Rev. D41 (1990) 3814.

[4] A.C. Tang, S.J. Brodsky, H.C. Pauli: Phys. Rev. D44 (1991) 1842.

[5] M. Kaluža, H.C. Pauli: Phys. Rev. D45 (1992) 2968; L.C.L. Hollenberg, K. Higashijima, R.C. Warner, B.H.J. McKellar: Prog. Theor. Phys. 87 (1992) 441.

[6] M. Krautgärtner, H.C. Pauli, F. Wölz: Phys. Rev. D45 (1992) 3755.

[7] D.G. Robertson: Phys. Rev. D47 (1993) 2549.

[8] T. Maskawa, K. Yamawaki: Prog. Theor. Phys. 56 (1976) 270.

[9] P.A.M. Dirac: Lectures on Quantum Mechanics. Yeshiva University, New York: Academic Press 1964; A. Hanson, T. Regge, C. Teitelboim: Constrained Hamiltonian Systems. Acc. Naz. dei Lincei 1976; K. Sundermeyer: Constrained Dynamics, Lecture Notes in Physics 169. Berlin, Heidelberg, New York: 1982.

[10] T. Heinzl, S. Krusche, E. Werner, B. Zellerman: University of Regensburg preprint TPR 92-17 1992.

[11] T. Heinzl, S. Krusche, S. Simbürger, E. Werner: Z. Phys. C - Particles and Fields 56 (1992) 415.

[12] C.M. Bender, S. Pinsky, B. van de Sande: Phys. Rev. D48 (1993) 816.

[13] G. McCartor, D.G. Robertson: Z. Phys. C - Particles and Fields 53 (1992) 679.

[14] M. Maeno: Osaka preprint OU-HET-175B, 1993.

[15] V.A. Franke, Y.V. Novozhilov, E.V. Prokhvatilov: Lett. Math. Phys. 5 (1981) 239; Lett. Math. Phys. 5 (1981) 437. 
[16] A.C. Kalloniatis, H.C. Pauli: Z.Phys. C - Particles and Fields 60 (1993) 255.

[17] A.C. Kalloniatis, H.C. Pauli, S.S. Pinsky: Dynamical Zero Modes and Pure Glue $Q C D_{1+1}$ in Light-Front Field Theory, Ohio State University preprint OHSTPY-HEPTH-93-017; A.C. Kalloniatis, H.C. Pauli, S.S. Pinsky: in preparation.

[18] F. Lenz, M. Thies, S. Levit, K. Yazaki: Ann. Phys. (N.Y.) 208 (1991) 1.

[19] N.S. Manton: Ann. Phys. (N.Y.) 159 (1985) 220; P. van Baal: Nucl. Phys. B 369 (1992) 259 , and references therein.

[20] G. Leibbrandt: Rev. Mod. Phys. 59 (1987) 1067; A. Bassetto, G. Nardelli, R. Soldati: Yang-Mills Theories in Algebraic Non-Covariant Gauges. Singapore: World Scientific 1991.

[21] S. Coleman: Ann. Phys. (N.Y.) 93 (1975) 267; 101 (1976) 239.

[22] G. McCartor: Z. Phys. C - Particles and Fields 52 (1991) 611; T. Heinzl, S. Krusche, E. Werner: Phys. Lett. B 256 (1991) 55.

[23] G. McCartor: Talk given at Third Meeting on Light-Cone Quantization and Nonperturbative Methods, PSI Zürich, June 1993.

[24] K.D. Rothe, H.J. Rothe, I.O. Stamatescu: Ann. Phys. (N.Y.) 105 (1977) 63.

[25] D.G. Robertson, private communication.

[26] F. Lenz, H.W.L. Naus, M. Thies: Erlangen preprint October 1993.

[27] H. Yabuki: Phys. Lett. B 231 (1989) 271.

[28] R.W. Brown, J.W. Jun, S.M. Shvartsman, C.S. Taylor: to appear in Phys. Rev. D (1993).

[29] A.C. Kalloniatis, D.G. Robertson: paper in preparation.

[30] C. Itzykson, J-B. Zuber: Quantum Field Theory. Singapore, McGraw-Hill: 1985. pp. $172-173$.

[31] J.B. Kogut, D.E. Soper: Phys. Rev. D1 (1970) 2901. 
MAX-PLANCK-INSTITUT FÜR KERNPHYSIK

SAUPFERCHECKWEG 1 - D-69117 HEIDELBERG POSTFACH 103980 • D-69029 HEIDELBERG

TELEFON 06221 - 5161 - TELEFAX $06221-516540$ 